\title{
DIRECT SUM PROPERTIES OF QUASI-INJECTIVE MODULES
}

BY K. R. GOODEARL

Communicated by Barbara Osofsky, October 15, 1975

\begin{abstract}
A functorial method is described by which certain problems can be transferred from quasi-injective modules to nonsingular injective modules. Applications include the uniqueness of $n$th roots: If $A$ and $B$ are quasi-injective modules such that $A^{n} \cong B^{n}$, then $A \cong B$.
\end{abstract}

All rings in this paper are associative with unit, all modules are unital right modules, and endomorphism rings act on the left. The letter $R$ denotes a ring. We use $J(-)$ to denote the Jacobson radical.

Recall that a module $A$ is quasi-injective provided any homomorphism of a submodule of $A$ into $A$ extends to an endomorphism of $A$. For example, all injective modules and all semisimple (completely reducible) modules are quasiinjective.

Theorem 1. Let $A$ be a quasi-injective right $R$-module, and set $Q=$ $\operatorname{End}_{R}(A)$. Then $Q / J(Q)$ is a regular, right self-injective ring, and idempotents can be lifted modulo $J(Q)$.

Proof. Regularity and idempotent-lifting were proved by Faith and Utumi [2, Theorems 3.1, 4.1]. Self-injectivity was proved by Osofsky [6, Theorem 12] and Renault [7, Corollaire 3.5]. 口

Proposition 2. Let $A$ be a quasi-injective right $R$-module, and set $Q=$ $\operatorname{End}_{R}(A)$. Let $\mathfrak{U}$ denote the category of all direct summands of finite direct sums of copies of $A$, and let $P$ denote the category of all finitely generated projective right $(Q / J(Q))$-modules. Then there exists an additive (covariant) functor $F: \mathbb{Q r} \rightarrow$ $P$ with the following properties.

(a) For all $B, C \in \mathbb{Q}$, the induced map $\operatorname{Hom}_{\mathfrak{x}}(B, C) \rightarrow \operatorname{Hom}_{p}(F(B), F(C))$ is surjective.

(b) Given any $P \in P$, there exists $B \in \mathcal{Q}$ such that $F(B) \cong P$.

(c) $A$ map $f \in \mathcal{U}$ is an isomorphism if and only if $F(f)$ is an isomorphism in $P$.

Proof. If $P_{0}$ denotes the category of all finitely generated projective right $Q$-modules, then $\operatorname{Hom}_{R}(A,-)$ defines a category equivalence $G: \mathfrak{U} \rightarrow P_{0}$. Second, $(-) \otimes_{Q}(Q / J(Q))$ gives us an additive functor $H: P_{0} \rightarrow P$, and we set $F=H G$.

AMS (MOS) subject classifications (1970). Primary 16A52. 
Properties (a) and (c) hold without any hypotheses on $A$, while (b) follows from the regularity of $Q / J(Q)$ and the fact that idempotents lift modulo $J(Q)$.

Over a regular, right self-injective ring, all finitely generated projective right modules are injective and nonsingular. Thus the functor $F$ in Proposition 2 enables us to transfer problems from the quasi-injective module $A$ to the nonsingular injective module $F(A)$.

TheOREm 3. Let $A, B$ be quasi-injective right $R$-modules, and let $n$ be a positive integer.

(a) If $A^{n}$ is isomorphic to a direct summand of $B^{n}$, then $A$ is isomorphic to a direct summand of $B$.

(b) If $A^{n} \cong B^{n}$, then $A \cong B$.

Proof. Setting $Q=\operatorname{End}_{R}(B)$, we use Proposition 2 to transfer the problem to nonsingular injective right $(Q / J(Q))$-modules, where the required properties follow from [5, Proposition 9.1]. 口

Definition. A module $A$ is directly finite provided $A$ is not isomorphic to any proper direct summand of itself.

THEOREM 4 [1, Proposition 5]. Let $A$ be a directly finite quasi-injective right $R$-module. If $B$ and $C$ are any right $R$-modules such that. $A \oplus B \cong A \oplus C$, then $B \cong C$.

PRoof. If $P$ is any directly finite nonsingular injective module, then [8, Corollary 8] (or [5, Theorem 3.8]) shows that isomorphic direct summands of $P$ have isomorphic complements. Using Proposition 2, the module $A$ has the same property. In addition, [3, Theorem 3] shows that $A$ has the exchange property, hence cancellation follows from [4, Theorem 2].

Corollary 5. If $A_{1}, \ldots, A_{n}$ are directly finite quasi-injective right $R$ modules, then $A_{1} \oplus \cdots \oplus A_{n}$ is directly finite (but not necessarily quasi-injective).

Proof. Obviously cancellation carries over from the $A_{i}$ to their direct sum. On the other hand, $\mathbf{Z} / 2 \mathbf{Z}$ and $\mathbf{Q}$ are directly finite quasi-injective $\mathbf{Z}$-modules whose direct sum is not quasi-injective.

THEOREM 6. If $A$ is a quasi-injective right $R$-module, then there exists a decomposition $A=B \oplus C$ such that $B$ is directly finite and $C \cong C^{2}$.

Proof. The corresponding decomposition for nonsingular injective modules is given by [5, Proposition 8.4 and Theorem 7.2]. 口

Corollary 7. Let $A$ be a quasi-injective right $R$-module. Then $A$ is directly finite if and only if $A$ has no nonzero direct summands $C$ for which $C \cong$ $C^{2}$. 口 


\section{REFERENCES}

1. G. F. Birkenmeier, On the cancellation of quasi-injective modules, Communications in Algebra (to appear)

2. C. Faith and Y. Utumi, Quasi-injective modules and their endomorphism rings, Arch. Math. 15 (1964), 166-174. MR 29 \#3503.

3. L. Fuchs, On quasi-injective modules, Ann. Scuola Norm. Sup. Pisa (3) 23 (1969), 541-546. MR 41 \#3518.

4. - The cancellation property for modules, Lectures on Rings and Modules (Tulane Univ. Ring and Operator Theory Year 1970-1971, Vol. I), Lecture Notes in Math., vol. 246, Springer-Verlag, Berlin, 1972, pp. 191-212. MR 49 \#2846.

5. K. R. Goodearl and A. K. Boyle, Dimension theory for nonsingular injective modules (to appear).

6. B. L. Osofsky, Endomorphism rings of quasi-injective modules, Canad. J. Math. 20 (1968), 895-903. MR 38 \#184.

7. G. Renault, Anneau associé à un module injectif, Bull. Sci. Math. (2) 92 (1968), 53-58. MR 38 \#1129.

8. Y. Suzuki, On automorphisms of an injective module, Proc. Japan Acad. 44 (1968), 120-124. MR 37 \#2806.

DEPARTMENT OF MATHEMATICS, UNIVERSITY OF UTAH, SALT LAKE CITY, UTAH 84112 